Preprint

UCRL-JC-138305

\title{
Acoustically-Driven Microfluidic Systems
}

\author{
A.W. Wang, E.J. Benett, L.R. Tarte \\ This article was submitted to International Mechanical Engineering \\ Conference \& Exposition, Orlando, FL, November 5-10, 2000
}

\section{June 23, 2000}

U.S. Department of Energy

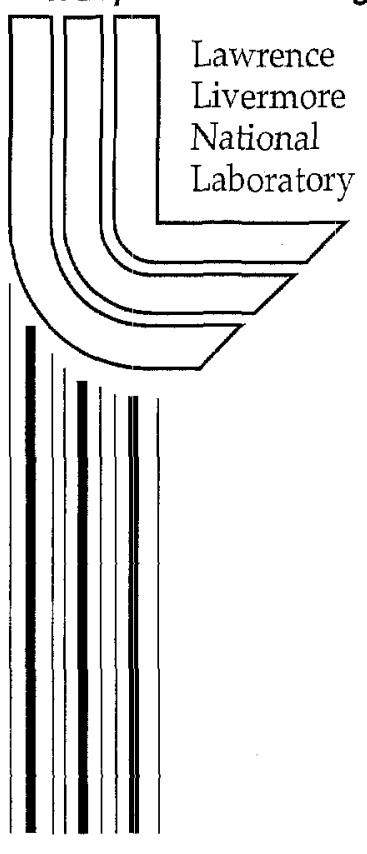




\section{DISCLAIMER}

This document was prepared as an account of work sponsored by an agency of the United States Government. Neither the United States Government nor the University of California nor any of their employees, makes any warranty, express or implied, or assumes any legal liability or responsibility for the accuracy, completeness, or usefulness of any information, apparatus, product; or process disclosed, or represents that its use would not infringe privately owned rights. Reference herein to any specific commercial product, process, or service by trade name, trademark, manufacturer, or otherwise, does not necessarily constitute or imply its endorsement, recommendation, or favoring by the United States Government or the University of California. The views and opinions of authors expressed herein do not necessarily state or reflect those of the United States Government or the University of California, and shall not be used for advertising or product endorsement purposes.

This is a preprint of a paper intended for publication in a journal or proceedings. Since changes may be made before publication, this preprint is made available with the understanding that it will not be cited or reproduced without the permission of the author.

This work was performed under the auspices of the United States Department of Energy by the University of California, Lawrence Livermore National Laboratory under contract No. W-7405-Eng-48.

This report has been reproduced directly from the best available copy.

Available electronically at http://www.doc.gov/bridge

Available for a processing fee to U.S. Department of Energy

And its contractors in paper from

U.S. Department of Energy

Office of Scientific and Technical Information

P.O. Box 62

Oak Ridge, TN 37831-0062

Telephone: (865) 576-8401

Facsimile: (865) 576-5728

E-mail: reports@adonis.osti.gov

Available for the sale to the public from

U.S. Department of Commerce

National Technical Information Service

5285 Port Royal Road

Springfield, VA 22161

Telephone: (800) 553-6847

Facsimile: (703) 605-6900

E-mail: orders@ntis.fedworld.gov

Online ordering: http://www.ntis.gov/ordering.htm

\section{OR}

Lawrence Livermore National Laboratory

Technical Information Department's Digital Library

http://www.llnl.gov/tid/Library.html 


\section{ACOUSTICALLY-DRIVEN MICROFLUIDIC SYSTEMS \\ Amy W. Wang, William J. Benett, Lisa R. Tarte \\ Lawrence Livermore National Laboratory}

\begin{abstract}
We have demonstrated a non-contact method of concentrating and mixing particles in a plastic microfluidic chamber employing acoustic radiation pressure. A flow cell package has also been designed that integrates liquid sample interconnects, electrical contacts and a removable sample chamber. Experiments were performed on 1, 3, 6, and $10 \mu \mathrm{m}$ polystyrene beads. Increased antibody binding to a solid-phase substrate was observed in the presence of acoustic mixing due to improve mass transport.
\end{abstract}

\section{Introduction}

Sample pre-treatment is one of the most critical aspects in the development of autonomous microfluidic systems, yet it remains largely an unsolved problem. A necessary goal of these systems is to obviate manual handling of samples, which is slow, requires trained personnel, and is not costeffective. Integrated sample preparation requires a high degree of functionality to truly permit biological or chemical analysis to be performed in a miniaturized system, yet the danger of sample-tosample contamination dictates inexpensive, disposable parts. Typically the cost of a part increases with its functionality.

We propose to use acoustic energy remotely coupled into a plastic chamber as a method of combining high functionality with an inexpensive, disposable sample chamber. Since the actuation method depends upon acoustic energy coupled in from an external source, integration of the piezoelectric transducers with the chamber is not required. Hence, plastic fluidic chambers can be manufactured inexpensively and thrown away after one use, while the piezoelectric transducers and associated electronics are reused. In addition, the electronics of acoustically-driven systems are isolated from the biological samples, reducing the likelihood of electrode fouling. This will increase the lifetime of the system, reduce maintenance, and permit continuous monitoring. The elimination of integrated components also allows the layout of the microfluidic system to be more easily reconfigurable and amenable to rapid prototyping.
Mixing in a biological laboratory typically is carried out on a shaker table or using a vortexer. Micro-scale mixing is a challenge since small channel dimensions make it difficult to create turbulence. Acoustic mixing brings with it the advantages of rapid mixing, no moving parts, and no need for external injection of fluids or nozzles to create turbulence. Previous work has shown an increased amount of antibody binding in the presence of acoustic mixing [1-2].

We use bulk piezoelectric tr.nsducers as an external source and couple the acoustic energy into plastic sample chambers. Distinct transport mechanisms can be activated by varying the orientation of the piezoelectric transducer and chamber configurations, or by changing the input energy levels. The primary acoustic transport principle we will employ is acoustic radiation pressure.

Acoustic radiation pressure exerts a force on a particle. Radiation pressure generated in the presence of a standing wave induces a pressure field that forces particles to collect in the nodes or anti-nodes of the standing wave. The acoustic force exerted on a particle can be expressed as,

$$
F_{a c}=-4 \pi / 3 R^{3} k E A \sin (2 k x),
$$

where $\mathrm{R}$ denotes the particle radius, $\mathrm{k}$ is the wave number, and $\mathrm{E}$ is the acoustic energy density [3-7]. The compressidensity factor, $A$, reflects the relative density and compressibility of the particle with respect to the ambient medium, and the sign of $A$ determines whether the particles concentrate in the nodes or the anti-nodes of the standing wave. It follows that acoustic radiation pressure can be used to differentiate particles based on their size and their material attributes.

\section{Experimental Set-up}

Removable acoustic source. The challenge for efficient acoustic coupling into a plastic, water-filled sample chamber is to maximize transmitted energy by matching the acoustic impedance from a relatively hard and dense piezoceramic, such as lead zirconate titanate (PZT), to the low acoustic impedance of plastics and water-like liquids. Typical methods employ either a horn-shaped structure or use a coupling layer to match impedance. Metal-seeded 
epoxies are often used as matching layers between PZT and fluids. To permit removal of the piezoceramic transducers from the sample container, rather than an epoxy, we use a silver-embedded elastomer as a matching material. We have found that this coupling layer provides sufficient acoustic coupling for particle manipulation functions of interest.

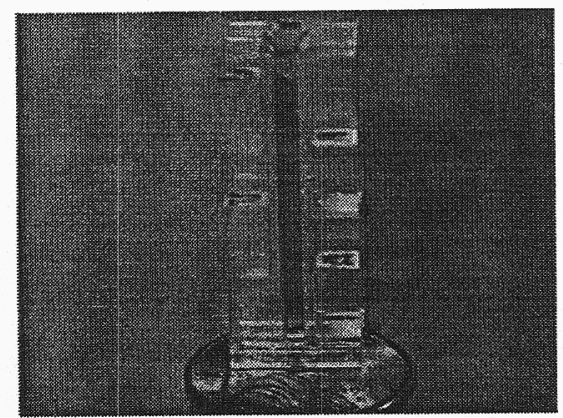

Fig. 1: Mixing sample chamber. Piezoceramic transducers are positioned down the length of the central fluid channel of the sample chamber.

Device fabrication and package. Fluid channels are laser cut in acrylic sheets and devices formed from a multi-layer, bonded laminate. We have developed two bonding processes, solvent bonding and UVcurable acrylic bonding. A flow cell package has been designed that integrates fluid interconnects developed by Benett et al. [8], electrical contacts to the piezoelectric transducers, and a removable sample chamber. Piezoelectric transducers are inserted into recessed pockets of the sample chamber. Electrical contact to the front and back face of the piezoceramic is made using conducting adhesive copper foil and conductive elastomer plugs. The elastomer plugs insert into a plastic fixture that mechanically clamps the piezoceramics in place. Electrical connectors are plugged into the fixture to contact the conducting elastomer. In this package format, use of a disposable sample chamber is possible.

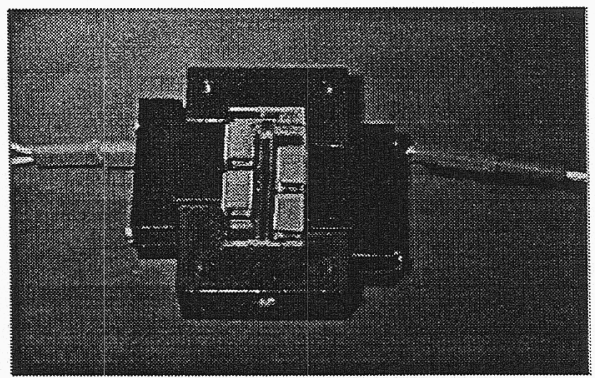

Fig. 2: Mixing device in package. Electrical interconnects are shown.
A mixing chamber is shown in Fig. 1, in which four piezoelectric (PZT) transducers are staggered down the length of a fluid channel. It was observed from early mixing experiments with single transducers that areas of high particle velocity occur at the corners of the piezoelectric transducers -- the transition area between a region of high pressure in front of the transducer and a region of low pressure where the acrylic wall is mechanically clamped. We consequently designed our chamber with periodic clamping of the fluid channel wall. Staggering the transducers permits a longer mixing length. The sample volume for this chamber was $200 \mu 1$.

Piezoelectric transducers are driven at 15-40 Vpp using a HP123 function generator and Krohn-hite amplifier ( $\mathrm{KC1}$ ). Characterization of the piezoelectric transducer resonance behavior was performed on a network analyzer (HP123). Optimal drive frequencies were also determined by measuring current through the transducer. A resonant peak of $355 \mathrm{kHz}$ was observed for a $4 \mathrm{~mm}$ thickness mode vibration. Pulsed drive voltages were also used to induce mixing in the channel. Sinusoidal amplitude modulation was used and the modulation frequency was varied to determine the effect on mixing. Degassing of the water was not required.

The efficacy of acoustic mixing was determined in mixed two-step immunoassay in which ovalbumincoated $5 \mu \mathrm{m}$ magnetic porous glass beads (Sigma) are bound with fluoroscein-labeled anti-ovalbumin. 100 $\mu \mathrm{g} / \mathrm{ml}$ ovalbumin was mixed in phosphate buffered saline and $0.1 \%$ Tween 20 . (The preparation) The effect of acoustic mixing on the amount of bound antibody was in determined using flow cytometry to determine the amount of fluorescent signal. 200 $\mathrm{\mu l}$ MPG-Ov solution was added to $100 \mu l \alpha$-Ov-FITC, and pippetted into the mixing chamber. Acoustic mixing was performed for five minutes in all experiments. The sample was then rinse $2 \mathrm{x}$ in PBSTween using a magnetic separator. The beads were then resuspended in $300 \mu l$ and analyzed on the flow cytometer.

\section{Results}

Acoustic Mixing. Results show we are able to induce acoustic mixing of glass and polystyrene beads down the full length of the fluid channel (Fig. 3). Bead mixing was achieved for $1,3,5,6$, and $10 \mu \mathrm{m}$ polystyrene beads and $5 \mu \mathrm{m}$ glass beads. Mixing 
behavior was observed at drive frequencies that correlated to observed piezoceramic resonance frequencies. Particle speed increased with drive voltage. Mixing stopped abruptly below $15 \mathrm{Vpp}$. Mixing plumes were observed directly in front of each piezoelectric transducer. Regions of highest particle velocity were observed in regions $a$ and $b$ of Fig. 2. Mixing along the completed channel length with a minimal number of piezoelectric transducers was achieved with a staggered transducer design.

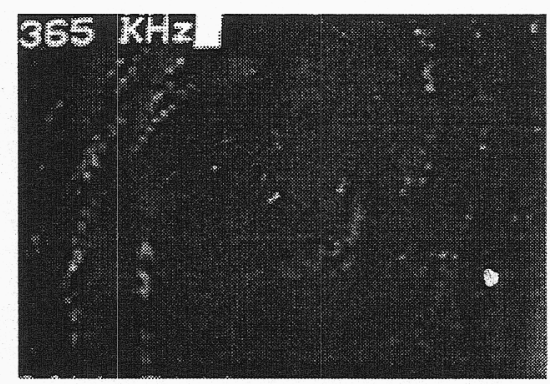

Fig. 3: Mixing of $10 \mu \mathrm{m}$ polystyrene beads in water.

The effect of acoustic mixing on binding kinetics was tested using ovalbumin binding to magnetic porous glass beads. Flow cytometry results represents an average fluorescent signal over 3000 beads. Percentage increase in binding and was calculated as compared to unmixed samples. Results showed an increased amount of ovalbumin binding in the presence of acoustic mixing compared with unmixed samples. The amount of bound ovalbumin increased with piezoelectric drive voltage as shown in Fig. 4. We conclude that the increased particle velocity associated with a higher drive voltage enhances mass transport and consequently accelerates binding kinetics.

Experiments in which the piezoelectric transducers

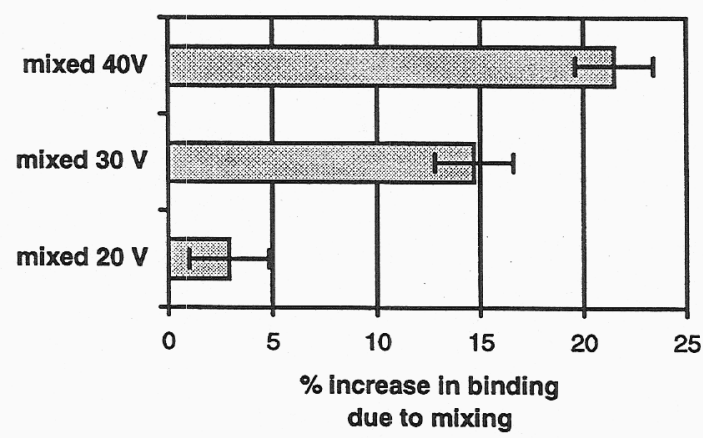

Fig. 4: Increased ovalbumin binding with increased piezoelectric drive voltage. A $0 \%$ increase indicates an unmixed sample. were driven at $40 \mathrm{Vpp}$ and amplitude modulated at varying frequency showed a dependence on the modulation frequency (Fig. 5). Faster modulation frequency appears to reduce the amount of bound ovalbumin. Similar detrimental effects of acoustic mixing has been reported previously [2]. In previously were it was observed that higher drive velocities resulted in less bound antibody on a solidphase substrate. It was hypothesized that the high

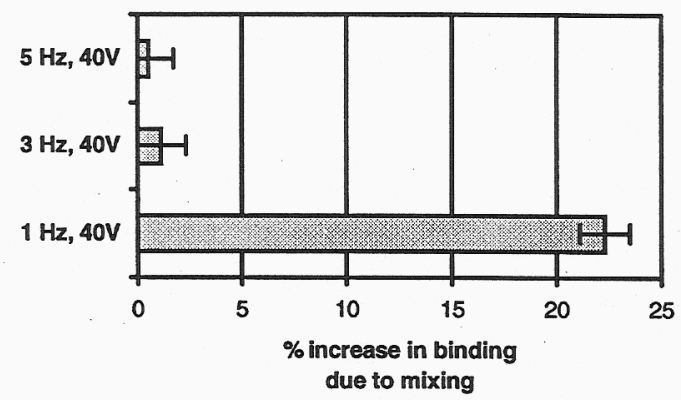

Fig. 5: Effect of pulsed mixing through amplitude modulation of the drive signal. Faster modulation frequency results in reduced ovalbumin binding.

shear forces generated at the substrate surface interfered with antibody binding. Videotape of particle behavior indicates a more continuous particle motion under slower modulation frequencies, as expected. We hypothesized that the more stuttered particle movement induced by higher modulation frequency results in less mass transport.

In order to understand better the observed mixing phenomenon, we performed finite element modeling of the fluid channel geometry. One-dimensional models verified generation of a standing pressure wave given the geometry of the chamber. Threedimensional models showed a distribution of pressure across the length of the fluid channel. Specifically, a higher pressure profile at the center of the wall, with decreasing energy radiating towards the channel ends. We believe this is due to the mechanical clamping at the ends of the acrylic wall, which appears to cause a circular mixing motion at the clamped wall interface.

Acoustic Concentration and Separation. Standing waves chambers were designed such that the width of the channel was equal to a half wavelength for the resonant drive frequency of the piezoelectric transducers. Driving the transducers 180 degrees out of phase results in a pressure node positioned at the center of the fluid channel. 
Concentration (Fig. 6) of polystyrene beads down the center of the channel was observed in water. Polystyrene $(p=1.05)$ is denser than water and half a compressor density factor equal to 0.7 ; hence, collection in the pressure node is expected.

Fig. 6 shows particle collection for piezoelectric transducers were driven at $20 \mathrm{Vpp}$. Bead concentration required a careful balance between the two transducers. At $430 \mathrm{kHz}$, bead concentration was observed after relative amplitude and frequency adjustment to the two transducers. An imbalance in radiation force resulted in mixing.

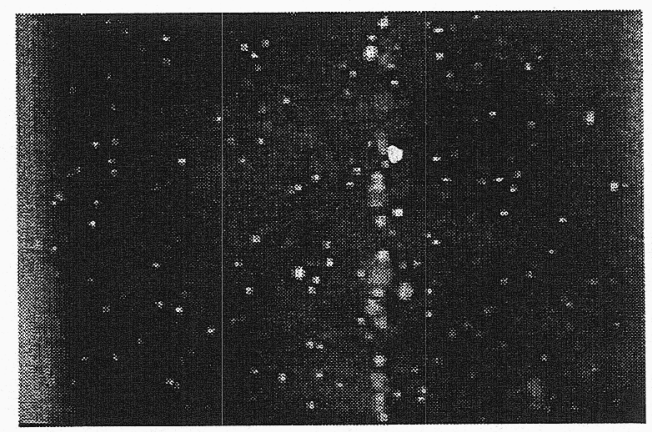

Fig. 6: Preferential polystyrene bead concentration.

Fig. 6 shows concentration of $10 \mu \mathrm{m}$ polystyrene beads at the center of the fluid channel, an expected pressure node of the standing wave. Also shown in Fig. 6 is separation of particles based on size. The larger beads collect at the central node of the channel, while smaller, $3 \mu \mathrm{m}$ beads remain dispersed in solution. Bead concentration times were on the order of minutes.

\section{Summary}

Mixing and concentration of particles in fluids has been shown in a plastic sample chamber with a flow cell package that permits reassembly and removal of the chamber. We have tested prototype designs that indicate preferential geometry for mixing versus concentrating functions. We have also developed a fabrication process suitable for large-feature channels using laser-cutting and solvent bonding of acrylic.

\section{Acknowledgements}

The authors gratefully acknowledge James Butler, Wayne Jensen for their laser cutting and plastics expertise, and Karl Fisher for frequent acoustics discussions.

\section{References}

1. R. Chen, L. Weng, N. C. Sizto, B. Oscorio, C. Hsu, R. Rodgers, D. J. Litman, "Ultrasonicaccelerated immunoassay, exemplified by enzyme immunoassay of choriogonadotropin," Clin. Chem., 30, 1446-1451, 1984.

2. A. W. Wang, R. Kiwan, .R. M. White, R. L. Ceriani, "A silicon-based ultrasonic immunoassay for detection of breast cancer antigens," Sensors \& Actuators, B, 49, 13-21, 1998.

3. L. V. King, Proc. Roy. Soc., A, 147. 212, 1934.

4. K. Yosioka, Y. Kawasima, "Acoustic Radiation Pressure on a compressible sphere," Acustica, 5, 167-173, 1955.

5. K. Yasuda, K Takeda, S. Umemura, "Studies on particle separation by acoustic radiation force and electrostatic force," Jpn. J. Appl. Phys., 35, 3295-3299, 1996.

6. Whitworth, M. A. Grundy, W. T. Coakley, "Transport and harvesting of suspended particles using modulated ultrasound," Ultrasonics, 29, 439-444, 1991.

7. A. H. Meng, A. W. Wang, R. M. White, "Ultrasonic Sample concentration for Microfluidic Systems," Proc. Tenth International Conference on Solid-State Sensors and Actuators, Sendai, Japan, June 1999.

8. W, H, Benett, P. Krulevitch, "Flexible packaging and interconnect scheme for microfluidic systems," Proc. Micro- and Nanofabricated Structures and Devices for Biomedical Environmental Applications, San Jose, CA, 111118, 1999. 